Journal of Alloys and Compounds, 615S1 (2014) 58-61

http://dx.doi.org/10.1016/j.jallcom.2013.11.118

\title{
Evolution of Fe environments in mechanically alloyed Fe-Nb-(B) compositions
}

J.S. Blázquez, J.J. Ipus, C.F. Conde, A.Conde

Departamento de Física de la Materia Condensada, ICMSE-CSIC, Universidad de Sevilla, P.O. Box 1065, 41080, Sevilla, Spain

\begin{abstract}
Nanocrystalline alloys of nominal composition $\mathrm{Fe}_{85} \mathrm{Nb}_{5} \mathrm{~B}_{10}$ were produced by mechanical alloying from a mixture of elemental powders. Two commercial boron structures were used: amorphous and crystalline. In addition, a third composition $\mathrm{Fe}_{94.4} \mathrm{Nb}_{5.6}$ was prepared for comparison. X-ray diffraction and Mössbauer spectroscopy were used to describe the evolution of the microstructure and $\mathrm{Fe}$ environments as a function of the milling time. Whereas $\mathrm{Nb}$ is rapidly incorporated into the nanocrystalline matrix, boron inclusions remain even after long milling times. The presence of boron is found to enhance the comminuting of crystallites.
\end{abstract}

Keywords: Mechanical alloying, Mössbauer spectroscopy, nanocrystalline alloys

Corresponding author: J. S. Blázquez

Tel: +34954556029

e-mail: jsebas@us.es 
Journal of Alloys and Compounds, $615 \mathrm{S1}$ (2014) 58-61

http://dx.doi.org/10.1016/j.jallcom.2013.11.118

\section{Introduction}

Nano-scale in Material Science has opened a wide research field in the last decades affecting numerous research areas. The decrease of the constituent units down to few nanometers leads to the appearance of new phenomenologies as the size of the characteristic correlation length is approached [1]. For example, coercivity decreases several orders of magnitude once the size of ferromagnetic crystallites falls below the exchange correlation length and thus magnetic domains comprise lot of crystallites leading to averaging out of the magnetocrystalline anisotropy [2].

Therefore, nanocrystalline systems have deserved the attention of the research community due to their soft magnetic properties [3]. The conventional way to produce them is by controlled crystallization of a precursor amorphous alloy produced by ultrarapid quenching. In order to diminish the cooling rate required to obtain the amorphous precursor alloy, metalloid elements, such as boron, are added to the master alloy to enhance the glass forming ability of the composition. On the other hand, mechanical alloying has been shown as a very effective tool to obtain these metastable structures in one step process [4]. However, it has been recently shown that pure boron inclusions remain even after long milling times [5].

In the present work, we study the evolution of the Fe environments during the nanocrystallization process of $\mathrm{Fe}_{94.4} \mathrm{Nb}_{5.6}$ and $\mathrm{Fe}_{90} \mathrm{Nb}_{5} \mathrm{~B}_{10}$ compositions induced by mechanical alloying, paying special attention to the incorporation of the different elements to the supersaturated solid solution matrix. Two different commercially available microstructures of the starting boron powder were used in order to search for the convenience of using one or the other.

\section{Experimental}

Elemental powder of nominal compositions $\mathrm{Fe}_{94.4} \mathrm{Nb}_{5.6}$ and $\mathrm{Fe}_{90} \mathrm{Nb}_{5} \mathrm{~B}_{10}$ (both with the same $\mathrm{Fe} / \mathrm{Nb}$ ratio) were ball milled in argon atmosphere using steel balls and hardened steel 
Journal of Alloys and Compounds, $615 \mathrm{S1}$ (2014) 58-61

http://dx.doi.org/10.1016/j.jallcom.2013.11.118

vials in a Fritsch Pulverisette Vario 4 planetary mill at $350 \mathrm{rpm}$ for the main disc and $-700 \mathrm{rpm}$ for the vials. Ball to powder ratio was 10:1. Two types of B-containing alloys were formed using two different commercial boron powders: crystalline and amorphous boron. Whereas the crystalline B powder is $\beta$-phase, the commercial amorphous B shows a $50 \%$ of amorphous phase [6]. In the following, they will be named as crystalline-B alloy and amorphous-B alloy, depending on the starting microstructure of used boron. X-ray diffraction (XRD) experiments were performed in a Bruker D8I diffractometer using $\mathrm{Cu}-\mathrm{K} \alpha$ radiation. Pawley method (full pattern fitting) [7] was used to fit the patterns of amorphous-B alloy and B-free alloy. XRD data of crystalline-B alloy were taken from a previous work [8]. Scanning electron microscopy (SEM) experiments were performed in a Jeol $6460 \mathrm{LV}$ microscope operated at $20 \mathrm{kV}$ equipped with energy dispersive X-ray (EDX) facility (Incax-sight Oxford Instruments). Mössbauer spectrometry (MS) experiments were performed in a transmission geometry using a ${ }^{57} \mathrm{Co}(\mathrm{Rh})$ source. Values of the hyperfine parameters were obtained by fitting with NORMOS program [9]. The isomer shift, $I S$, was quoted relative to the Mössbauer spectrum of an $\alpha-\mathrm{Fe}$ foil at room temperature. Two hyperfine field $(H F)$ distributions were used to fit the spectra (from 0 to $14 \mathrm{~T}$ and from 8 to $39 \mathrm{~T}$ ) to consider the possible different nature of the low and high field contributions. Quadrupolar splitting was set to zero assuming spherical symmetry of the environments.

\section{Results and discussion}

Figure 1 shows the XRD patterns of the samples after different times of milling. The main diffraction maxima appearing in the patterns are those of bcc $\alpha$-Fe phase. $\mathrm{Nb}$ lines are also observed at short milling times. As milling progresses the bcc-Fe maxima shift to lower angles and broaden for all the studied alloys. The shift to lower angles is related to an increase of lattice parameter, $a$, (see figure $2 \mathrm{a}$ ) and is common for the three studied alloys. This should indicate a negligible effect of $\mathrm{B}$, which incorporation to bcc Fe should lead to a decrease in $a$. In 
Journal of Alloys and Compounds, $615 \mathrm{S1}$ (2014) 58-61

http://dx.doi.org/10.1016/j.jallcom.2013.11.118

fact, as studied by Ray and Hasegawa for rapidly quenched FeB alloys, there are two boron atoms as substitutional in bcc Fe per each interstitial $\mathrm{B}$ atom and thus leading to a predominant substitutional role for B and to a decrease of the lattice parameter of bcc $\mathrm{Fe}|\Delta \mathrm{a}|<0.001 \AA$ per at. $\%$ of $\mathrm{B}$ [10]. Therefore, incorporation of $\mathrm{Nb}$ to the bcc Fe nanocrystals should explain the increase in $a$. Figure $2 \mathrm{a}$ also shows the $\mathrm{Nb}$ content that should correspond to each value of the lattice parameter following the Vegard law (right axis). Increase in $a$ value saturates after $4 \mathrm{~h}$, indicating that, after this milling time, $\mathrm{Nb}$ incorporation to bcc nanocrystals can be considered as a completed process. However, at milling times longer than $40 \mathrm{~h}$ for B-free alloy, a slight decrease in $a$ is observed, which could be ascribed to an enrichment in Fe due to contamination from the milling media. Unlike angular shift, broadening of the diffraction maxima only saturates for B-free alloy after $4 \mathrm{~h}$ milling, whereas B-containing alloys show a continuous trend up to the maximum explored milling time. This broadening is ascribed to crystal size reduction and microstrains increase. Concerning microstrains, $\varepsilon$, (figure $2 b$ ), there is no significant change among the studied alloys and its value is almost stabilized after $4 \mathrm{~h}$, as it occurs for the lattice parameter. However, crystal size, $D$, (figure 2 c) is clearly lower for B-containing alloys. It is worth mentioning that, for crystalline-B alloy, XRD data have been taken from our previous data reported in Ref. [8], where a single peak fitting was performed. In that fitting, the complete width of the diffraction maxima was independently ascribed to crystal size or microstrains, leading to limit values of maximum $\varepsilon$ and minimum $D$. This explains the absence of saturation in $\varepsilon$ as well as the smaller $D$ values for crystalline-B samples with respect to amorphous-B samples. Moreover, the single peak fitting is the responsible for the larger error bars shown in figure $2 \mathrm{a}$ for the lattice parameter.

Hyperfine field distributions obtained from the fitting of Mössbauer spectra are shown in figure 3. As milling progresses, the main feature observed is the broadening of the high field peak and its shift to lower $H F$ values. Both effects are enhanced in B-containing alloys with respect to B-free alloy. For the latter alloy, the evolution stabilizes after $4 \mathrm{~h}$ milling, in agreement with XRD results described above and ascribed to the progressive incorporation of 
Journal of Alloys and Compounds, $615 \mathrm{S1}$ (2014) 58-61

http://dx.doi.org/10.1016/j.jallcom.2013.11.118

$\mathrm{Nb}$ into the bec supersaturated solid solution and the stabilization of the size of nanocrystals. A priori, these two effects should be also responsible for the evolution observed in B-containing alloys. In fact, B incorporation to the matrix is not very effective and boron inclusions remains even after long milling times. These inclusions have been observed by transmission electron microscopy in B-crystalline alloy [5]. In figure 4, back scattering SEM images of amorphous-B samples milled for different times are shown. B inclusions are identified as dark spots, which are dispersed throughout the sample (see EDX in Fig. 4). The size of these inclusions decreases as milling time increases, indicating a progressive degradation of boron inclusions. Therefore, boron must be partially but slowly incorporated to the matrix. As B effect on crystalline phase is not detected through XRD data, it is inferred that this element should preferentially partition to the grain boundary region.

Further discussion on Mössbauer data yields the results shown in figure 5, where low field contributions $(H F<5 \mathrm{~T})$, average hyperfine field, $<H F>$, and average isomer shift, $\langle I S>$, are shown. The former parameter should correspond to Fe atoms in poor Fe environments. It is worth noticing that the evolution of $H F<5 \mathrm{~T}$ contributions is similar for the studied alloys and a maximum is achieved at very low milling times $(2.5-3 \mathrm{~h})$ reaching a constant value after $6 \mathrm{~h}$. Therefore, this dependency must be ascribed to $\mathrm{Nb}$ incorporation into the matrix. The maximum for $H F<5 \mathrm{~T}$ contribution should correspond to the milling time at which the highly deformed $\mathrm{Nb}$ crystals exhibit a maximum in their surface to volume ratio, leading to a maximum fraction of $\mathrm{Fe}$ atoms facing these $\mathrm{Nb}$-rich regions. As milling progresses, $\mathrm{Nb}$ atoms become dissolved and dispersed in the matrix and the probability for enriched $\mathrm{Nb}$ environments decreases in agreement with the observed trend for $H F<5 \mathrm{~T}$ contributions. Evolution of $<I S>$ with milling time is also similar for the three studied compositions and thus it must be ascribed to $\mathrm{Nb}$ effect. The large error of this parameter $( \pm 0.01 \mathrm{~mm} / \mathrm{s})$ prevents further discussion on it.

Unlike both $H F<5 \mathrm{~T}$ contributions and $\langle I S>$ parameters, which evolution is independent of B-content, there are clear differences between $<H F>$ evolution for B-containing and B-free alloys. Whereas for the latter alloy this parameter is almost stabilized after $4 \mathrm{~h}$ 
Journal of Alloys and Compounds, 615S1 (2014) 58-61

http://dx.doi.org/10.1016/j.jallcom.2013.11.118

milling, a continuous decrease is observed for both B-containing alloys. This continuous decrease should be ascribed to the slow incorporation of B to the matrix but also to the observed reduction of the crystal size. In fact, as $D$ decreases, the fraction of the so-called interface region (with a lower average $H F$ than that corresponding to the core [11]) is enhanced, and a sum rule could thus explain the continuous decrease observed in $\langle H F>$.

\section{Conclusions}

The evolution of $\mathrm{Fe}$ environments during mechanical alloying of $\mathrm{Fe}_{85} \mathrm{Nb}_{5} \mathrm{~B}_{10}$ and $\mathrm{Fe}_{94.4} \mathrm{Nb}_{5.6}$ compositions is affected by the incorporation to a bcc Fe-type matrix of $\mathrm{Nb}$ and $\mathrm{B}$ atoms. In a first stage, which saturates at $\sim 4 \mathrm{~h}$ milling in our experiments, $\mathrm{Nb}$ is incorporated to the bcc matrix. XRD results have shown that this element is effectively incorporated into the bcc crystals forming a supersaturated solid solution. However, B inclusions remain even at the end of the explored milling range, although boron inclusions are progressively reduced in size. The slow integration of B into the matrix shows no clear effect on the crystalline bcc phase. This fact implies that B atoms should preferential partition to the grain boundaries.

The presence of boron (independently of its initial microstructure) is shown to be very effective in reducing the crystallite size of the bcc phase. The continuous decrease of the crystal size observed in B-containing alloys should be the responsible for the continuous decrease observed in the average hyperfine field of these two alloys, whereas for B-free alloy both magnitudes, $D$ and $\langle H F>$, are stabilized after $6 \mathrm{~h}$ of milling.

\section{Acknowledgements}

This work was supported by the Spanish Ministry of Science and Innovation and EU FEDER (Project MAT 2010-20537) and the PAI of the Regional Government of Andalucía (Project P10-FQM-6462). 
Journal of Alloys and Compounds, 615S1 (2014) 58-61

http://dx.doi.org/10.1016/j.jallcom.2013.11.118 
Journal of Alloys and Compounds, 615S1 (2014) 58-61

http://dx.doi.org/10.1016/j.jallcom.2013.11.118

\section{Figure captions}

Figure 1. XRD patterns of samples milled different selected times.

Figure 2. (a) Lattice parameter (left axis) and estimated $\mathrm{Nb}$ content (right axist), (b) microstrains and (c) crystal size vs. milling time.

Figure 3. Hyperfine field distributions obtained for the different milled samples.

Figure 4. Back scattered scanning electron images of a $10 \mu \mathrm{m}^{2}$ area of amorphous-B alloy samples after different milling times. Top frame corresponds to EDX spectrum on a dark spot for the sample milled $0.5 \mathrm{~h}$ showing the emission peaks of $\mathrm{B}, \mathrm{Fe}$ and $\mathrm{Nb}$.

Figure 5. Low hyperfine field contribution (with $H F<5 \mathrm{~T}$ ), average hyperfine field and average isomer shift for the three studied alloys as a function of the milling time. 
Journal of Alloys and Compounds, 615S1 (2014) 58-61

http://dx.doi.org/10.1016/j.jallcom.2013.11.118

\section{Figure 1}

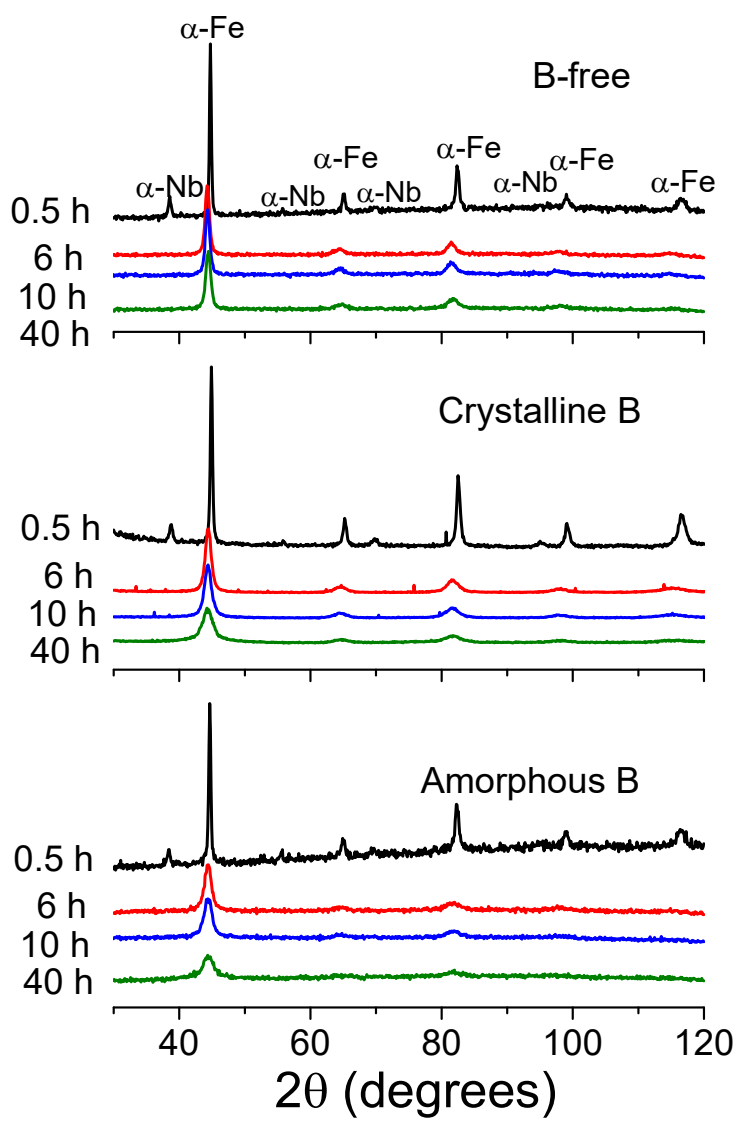


Journal of Alloys and Compounds, 615S1 (2014) 58-61

http://dx.doi.org/10.1016/j.jallcom.2013.11.118

\section{Figure 2}

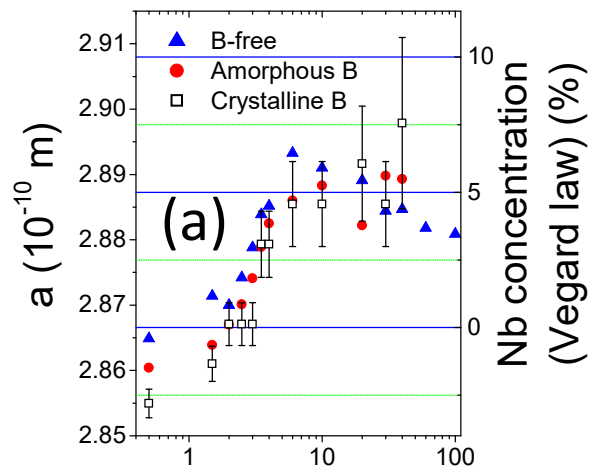

milling time $(\mathrm{h})$

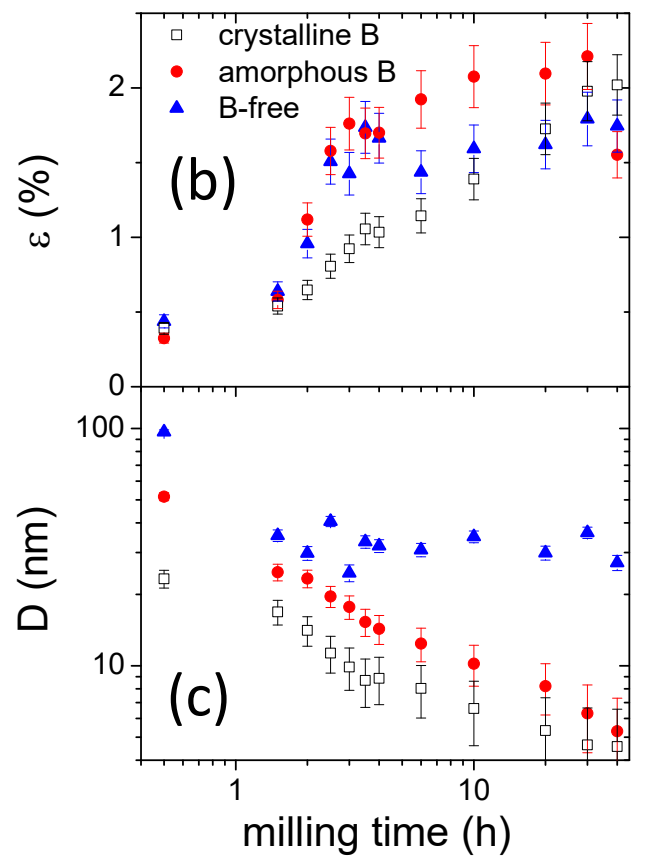


Journal of Alloys and Compounds, 615S1 (2014) 58-61

http://dx.doi.org/10.1016/j.jallcom.2013.11.118

\section{Figure 3}

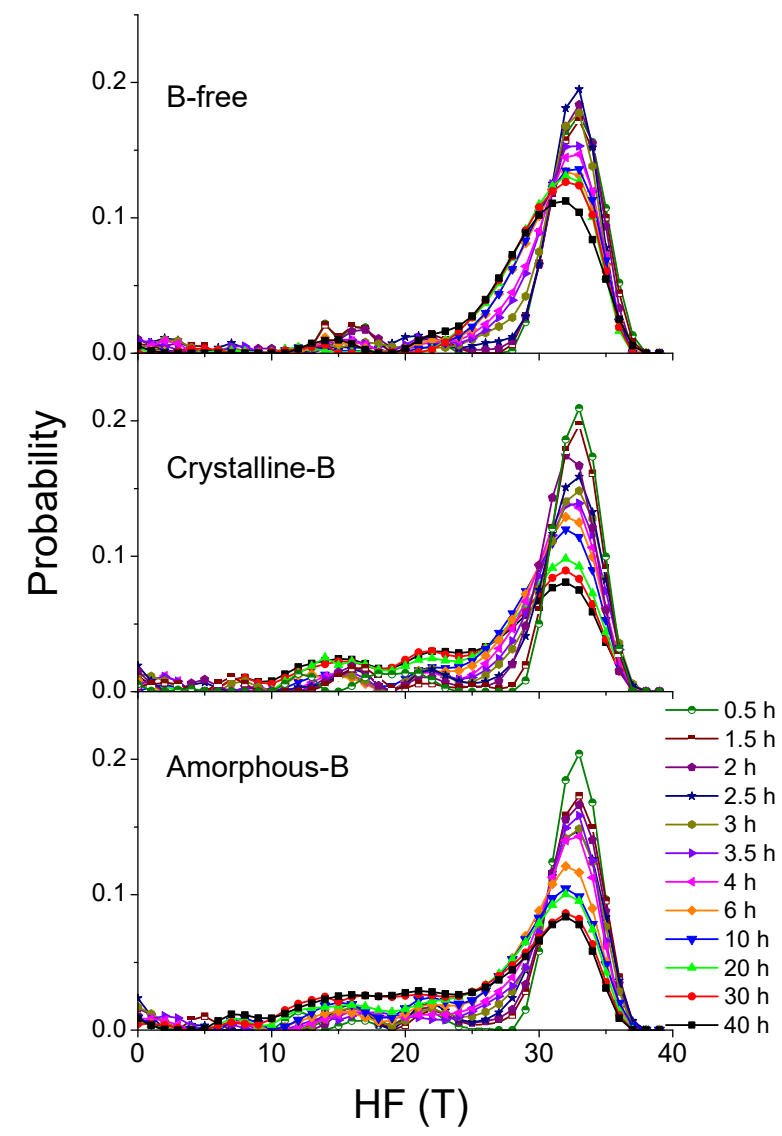


Journal of Alloys and Compounds, 615S1 (2014) 58-61

http://dx.doi.org/10.1016/j.jallcom.2013.11.118

\section{Figure 4}

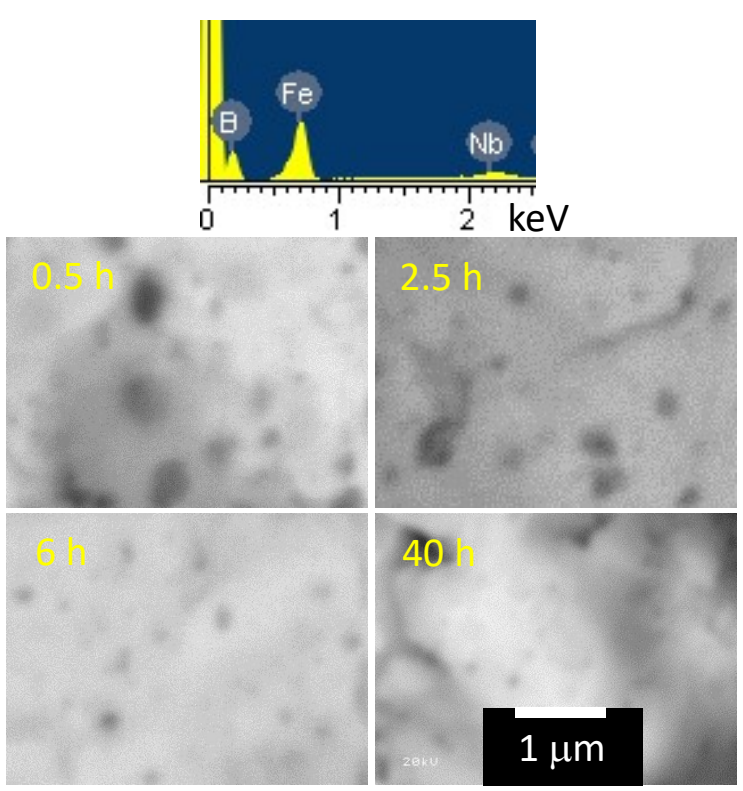


Journal of Alloys and Compounds, 615S1 (2014) 58-61

http://dx.doi.org/10.1016/j.jallcom.2013.11.118

\section{Figure 5}

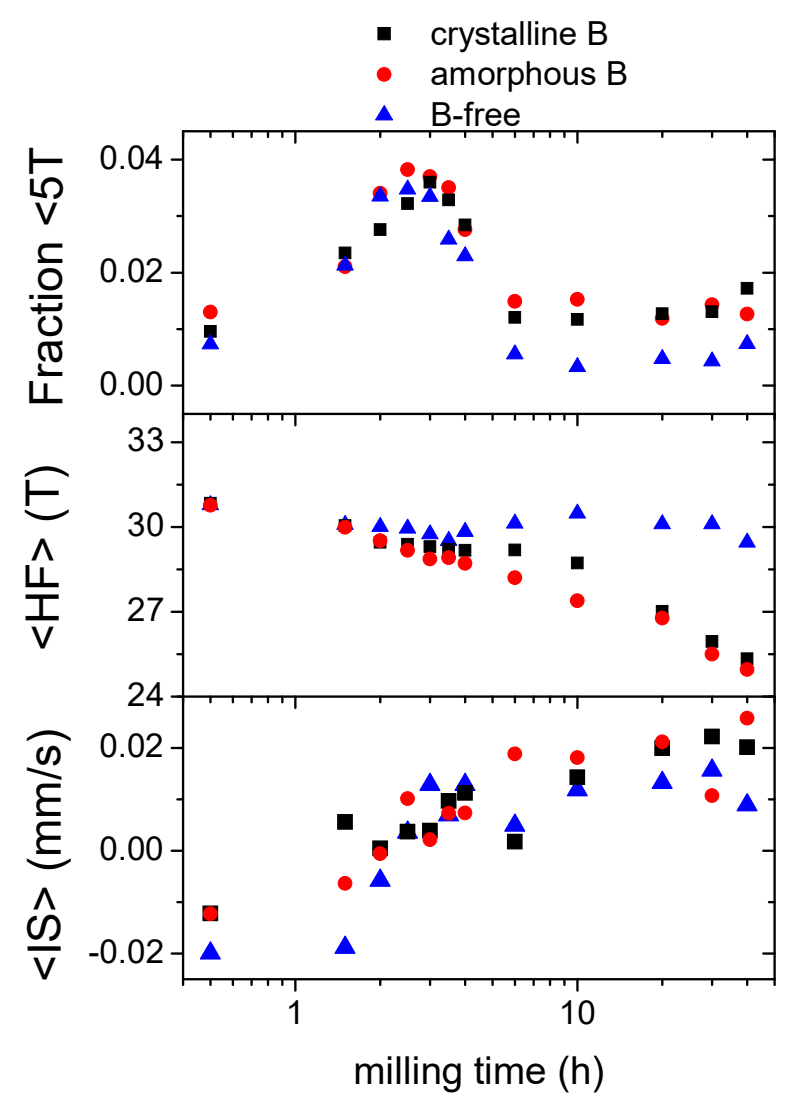


Journal of Alloys and Compounds, 615S1 (2014) 58-61

http://dx.doi.org/10.1016/j.jallcom.2013.11.118

\section{References}

[1] H. Gleiter, Prog. Mater. Sci. 33 (1989) 223

[2] G. Herzer, IEEE Trans. Mag. 26 (1990) 1397

[3] M.E. McHenry, M. A. Willard, D.E. Laughlin, Prog. Mater. Sci. 44 (1999) 291

[4] C. Suryanarayana, Prog. Mater. Sci. 46 (2001) 1

[5] J.J. Ipus, J.S. Blázquez, S. Lozano-Pérez, A. Conde, Phil. Mag. 89 (2009) 1415

[6] J.J. Ipus, J.S. Blázquez, V. Franco, S. Lozano-Pérez, A. Conde, J. All. Compd. 553 (2013) 119

[7] G.S.Pawley, J. Appl. Cryst. 13 (1980) 630.

[8]J.J. Ipus, J.S. Blázquez, V. Franco, A. Conde, Intermetallics, 16 (2008) 1073

[9] R. A. Brand, J. Lauer, D. M. Herlach, J. Phys. F: Met. Phys. 13 (1983) 675

[10] R. Ray, R. Hasegawa, Solid State Comm. 27 (1978) 471

[11] J. M. Greneche, Hyperfine Interactions 110 (1997) 81 